\title{
fMRI: blood oxygen level-dependent activation during a working memory-selective attention task in children born extremely preterm
}

\author{
Silja Torvik Griffiths', Hilde Gundersen², Emanuel Neto', Irene Elgen'1, Trond Markestad', Stein M. Aukland \\ and Kenneth Hugdahl ${ }^{1,4}$
}

\begin{abstract}
BACKGROUND: Extremely preterm (EPT)/extremely low-birth-weight (ELBW) children attaining school age and adolescence often have problems with executive functions such as working memory and selective attention. Our aim was to investigate a hypothesized difference in blood oxygen level-dependent (BOLD) activation during a selective attention-working memory task in EPT/ELBW children as compared with term-born controls.
\end{abstract}

METHODS: A regional cohort of 28 EPT/ELBW children and 28 term-born controls underwent functional magnetic resonance imaging (fMRI) scanning at $11 \mathrm{y}$ of age while performing a combined Stroop n-back task. Group differences in BOLD activation were analyzed with Statistical Parametric Mapping 8 analysis software package, and reaction times (RTs) and response accuracy (RA) were compared in a multifactorial ANOVA test.

RESULTS: The BOLD activation pattern in the preterm group involved the same areas (cingulate, prefrontal, and parietal cortexes), but all areas displayed significantly less activation than those in the control group, particularly when the cognitive load was increased. The RA results corresponded with the activation data in that the preterm group had significantly fewer correct responses. No group difference was found regarding RTs.

CONCLUSION: Children born EPT/ELBW displayed reduced working memory and selective attention capacity as compared with term-born controls. These impairments had neuronal correlates with reduced BOLD activation in areas responsible for online stimulus monitoring, working memory, and cognitive control.

A $\mathrm{n}$ increasing number of extremely preterm (EPT)/ extremely low-birth-weight (ELBW) children survive due to recent advances in neonatal medicine (1), but new challenges emerge as they attain school age. Even apparently well-functioning EPT children within the normal range of cognitive function often experience learning difficulties and are less likely to complete higher education than their term peers $(2,3)$. Recent studies indicate that several preterm children suffer from attention, concentration, and learning difficulties (4-7).

Impairment of cognitive functions such as working memory and selective attention has been confirmed in preterm children by several authors $(8-10)$. Working memory can be considered a "hub" function or connection that is central when it comes to cognitive capacity and is thus of crucial importance when investigating cognitive function $(11,12)$. Similarly, selective attention in the sense of being able to shift attention from one focus to another is an equally crucial aspect of normal cognitive functioning. The challenges preterm children meet in everyday life are rarely single-cognitive functions, rather the combined effects of several functions operating in parallel. Tapping only a single function as in most studies therefore limits possible conclusions. The present study included working memory and selective attention in the same task, allowing for evaluation of the combined effects of being born preterm on combined higher cognitive functions.

Some reports suggest that neurodevelopmental impairment following preterm birth may represent deficits in neural networks or development of compensatory networks, as reflected in the pattern of brain activation seen in some preterm children as compared with full-term controls $(13,14)$. Functional magnetic resonance imaging (fMRI) has revealed differences in neuronal brain activation between preterm and term children for a range of processes such as memory, language, and auditory measures (15-17). A common feature of these studies is that preterm children appear to mostly use the same neuronal pathways as term children, but they display significantly reduced magnitudes of activation. This could possibly indicate a developmental lag in brain maturation, or alternatively, a sign of different pathways between brain structures (often referred to as alternative pathways), leading to different activation patterns (16). The theory of alternative pathways has been supported by diffusion tensor imaging data (18). To our knowledge, all previous fMRI studies in this field involved a combination

\footnotetext{
'Department of Clinical Medicine, University of Bergen, Bergen, Norway; ${ }^{2}$ Department of Public Health and Primary Health Care, University of Bergen, Bergen, Norway; ${ }^{3}$ Department of Clinical Science, University of Bergen, Bergen, Norway; ${ }^{4}$ Division of Psychiatry, Haukeland University Hospital, Bergen, Norway. Correspondence: Silja Torvik Griffiths (silja.torvik.griffiths@helse-bergen.no)
} 
of EPT and more mature preterm children, the number of participants was often small, and none of the cohort samples were population based. Because recent research has provided more knowledge regarding the functional pathways of working memory and selective attention, which are particularly challenging functions to preterm children, it is important to investigate potential differences in activation patterns between EPT/ELBW and term-born children during a selective attention and working memory task (see ref. 19 for review).

We used fMRI and blood oxygen level-dependent (BOLD) contrast to study activation patterns while the children performed a combination of the Stroop color-word task and an n-back working memory task, which combines an assessment of selective attention and working memory.

The aims of the study were to evaluate (i) whether EPT/ ELBW children show different patterns of neuronal brain activation as compared with term-born peers, particularly regarding the involvement of prefrontal areas and the anterior cingulate cortex (ACC), which has been reported to be involved in processes related to selective attention and working memory (20); (ii) whether a difference in activation corresponds to differences in response accuracy (RA) and/or reaction time (RT); and (iii) whether any group difference increases with increasing cognitive load.

\section{RESULTS}

\section{Participants}

Thirty-six preterm children and 36 controls were scanned, but 8 preterm children and 8 controls had to be excluded due to head movement artifacts $(>5 \mathrm{~mm}$ translation and rotation movements). The final sample thus consisted of 28 (70\%) EPT/ ELBW children and 28 term-born controls (Table 1).

There was no statistically significant difference between the 12 excluded EPT/ELBW children (8 for movement artifacts plus 4 for reasons given in the Methods section) and the 28 included in analyses regarding gender, handedness, gestational age, birth weight, degree of neurological disability, or level of mother's education.

Table 1. Subject description

\begin{tabular}{lcc}
\hline Group & $\begin{array}{c}\text { Preterm group } \\
(n=28)\end{array}$ & $\begin{array}{c}\text { Control group } \\
(n=28)\end{array}$ \\
\hline Boys $^{\mathrm{a}}$ & $15(54)$ & $16(57)$ \\
$\begin{array}{l}\text { Birth weight, } \mathrm{g}^{\mathrm{b}} \\
\text { Gestational age, wk }\end{array}$ & $898( \pm 154)$ & $3,737( \pm 413)$ \\
$\begin{array}{l}\text { Periventricular } \\
\text { hemorrhage (mild degree) }\end{array}$ & $27( \pm 1.3)$ & $>37$ \\
$\begin{array}{l}\text { Left handedness } \\
\text { a }\end{array}$ & $4(14)$ & - \\
$\begin{array}{l}\text { Mother's education level } \leq \\
\text { secondary school }\end{array}$ & $3(11)$ & $3(11)$ \\
$\begin{array}{l}\text { Neurodevelopmental } \\
\text { impairments }\end{array}$ & $2(7)$ & $1(3)$ \\
\hline
\end{tabular}

${ }^{a}$ Number (\%). bMean (SD). c Cerebral palsy, mental retardation, or severe sensory deficits.

\section{fMRI Activation Patterns}

Main areas of activation. The overall pattern of BOLD activation in the four conditions (word 1-back, word 2-back, color 1-back, and color 2-back) revealed reduced activation in the preterm group as compared with the control group, but the pattern of activation involved the same areas Table 2 and Figure 1.

Areas of activation were seen bilaterally in the occipital inferior gyrus (Brodmann area (BA) 18/19), the supplementary motor area on the right side (BA 6), bilaterally in an area extending from the ACC (BA 24/32) to the middle frontal and precentral gyrus (BA 6/44/45), bilaterally in insula (BA 47/48), and bilaterally in the angular area extending into the parietal superior and inferior gyri (BA 7/40). The ACC, prefrontal, and parietal areas showed more activation in the 2-back conditions than in the 1-back conditions, indicating increased processing demands in the 2 -back conditions. Figure 1 and corresponding Table 2 describe main effects for the different experimental conditions, split for the preterm and control groups. Corresponding Montreal Neurological Institute (MNI, Canada) template coordinates for peak voxel activations for the significant clusters are given in Table 2 .

Word 1-back. Subtracting BOLD activation of the preterm from the control group in the word 1-back condition yielded activation in the supplementary motor area on the right side (BA 6), left ACC (BA 24), and the left calcarine sulcus (BA 17).

Color 1-back. Subtracting BOLD activation of the preterm from the control group in the color 1-back condition yielded activation in the left calcarine sulcus (BA 17), bilateral activation in the middle occipital region (BA 19), activation in the right supplementary motor area and precentral area (BA 6), activation in the ACC (BA 32), and bilateral activation in the insula (BA 47/48).

Word 2-back. Subtracting BOLD activation of the preterm from the control group in the word 2-back condition yielded activation only in the left middle occipital region (BA 19).

Color 2-back. Subtracting BOLD activation of the preterm from the control group in the color 2-back condition yielded bilateral activation in the middle occipital region (BA 19), activation in the right supplementary motor area (BA 6), bilateral activation in the insula (BA 47/48), bilateral activation in the frontal supplementary area (BA 6/45/47), and activation in the right lingual area (BA 19).

The opposite subtractions, controls minus preterms, showed no remaining activation in any of the four tasks.

To sum up this section, we found significantly more activation in the control group than in the preterm group, mainly in the color 1-back and 2-back conditions. Applying a family-wise error-corrected significance threshold showed no remaining significances. As a check of the robustness of the group-difference activations, we also ran the analyses with a false-discovery rate-corrected $(P<0.05)$ significance threshold. This showed 


\section{Articles | Grifiths et al.}

similar remaining activations for the main effect of group across conditions. When we separated the between-group comparisons for each of the stimulus conditions, there were remaining activations for the controls in the color 1-back condition.

\section{Region of Interest Analysis}

A region of interest (ROI) analysis of the ACC revealed less activation in dorsal ACC in the preterm group as compared with the control group in all the four research conditions $(P<$ 0.001 ; uncorrected, minimum cluster size 10 voxels). For the word 1-back and 2-back conditions, the main area was in BA 32; for the color 2-back condition a larger area also covered BA 24. The preterm group, however, showed no clusters of more than 10 voxels in any of the 1-back conditions. For the color and word 2-back conditions, we found activation in the dorsal ACC also in this group, but subtracting the BOLD activation of the control group from the preterm group left no cluster of more than 10 voxels in any conditions for the preterm group. Subtraction analyses reveal significant clusters of activation in both color conditions (BA 24/32) in the control group that could not be found in the preterm group, indicating that the color conditions were the main sites of group difference.

\section{Tests for Equality of Variances in the Two Groups}

Given the intraindividual variability in the BOLD signal in the preterm children across tasks, one could suspect that the BOLD responses in the preterm children are not normally distributed as compared with those of the control children. To test this, we extracted the BOLD signal across a spherical region with a $5-\mathrm{mm}$ radius around the peak voxel for each subject in both the groups in selected ROIs. From the group analysis, three ROIs were chosen as representative of overlapping activations between groups (at Montreal Neurological Institute template coordinates (33, $-85,-5)$ occipital middle (L), $(-21,-91,3)$ occipital inferior $(\mathrm{R})$, and $(5,19,51)$ supplementary motor $(\mathrm{R})$, respectively). Using inhouse scripts built around functions in the Statistical Parametric Mapping 8 (SPM8) software package (Welcome Trust Centre for Neuroimaging, London, UK), intensity within the ROI was averaged throughout the volume on spatially normalized frames for each individual subject and then averaged along the time course grouped by on/off blocks. The relative change in intensity was calculated according to the formula (on/off) -1 . We then tested the differences in homogeneity of variance/covariance matrices using Levene's test (21). The results of the test showed nonsignificant $(P>0.05)$ differences between the groups for all three ROIs. We therefore conclude that comparing the premature and the control groups using a general linear model approach, as implemented in SPM8, does not contain any violation of the assumption of equality of variance between the groups. Figure 2 shows probability-probability plots revealing the overlap between expected normal values and the observed values for the preterm children and the controls.

\section{Task Performance}

Response accuracy. The three-way ANOVA yielded significant main effects of group, $\mathrm{F}(1,54)=6.00, P<0.02$, with the preterm group having fewer correct responses than the control group, and of load, F $(1,54)=68.05, P<0.001$, with overall reduced RA for the 2-back as compared with the 1-back condition. There was no significant difference in RA for the instruction factor. The group $\times$ load interaction, however, was significant, F $(1,54)=6.56, P<0.02$, and a post hoc Tukey honestly significant difference test revealed that the reduced RA for the preterm group as compared with the control group occurred mainly for the color 2-back condition $(P<0.05)$ and was borderline, but not significant, for the word 2-back condition $(P=$ 0.060). The RA results are graphically shown in Figure 3.

Reaction time. The three-way ANOVA showed significant main effects for the factors load, $\mathrm{F}(1,54)=9.097, P<0.005$, and instruction, $\mathrm{F}(1,54)=7.75, P<0.01$, showing longer RTs for the 2-back as compared with the 1-back condition, and for processing of the ink color as compared with the word for the participants taken as a whole. There was no significant group effect $\mathrm{F}(1,54)=3.27, P=0.076$, only a trend toward longer RTs for the preterm group as compared with the control group Figure 4.

Cognitive load. As displayed in the results above, the group difference in BOLD activation patterns was increased in the color 1- and 2-back conditions relative to the other conditions, consistent with the largest group difference in RA being in the color 2-back condition. In other words, the preterm children managed the simple tasks but made more mistakes when the cognitive load was increased.

\section{DISCUSSION}

In this regional cohort study we found that 11-y-old EPT/ ELBW children as compared with term-born controls showed overall significantly reduced BOLD activation when performing a working memory-selective attention task, particularly in the more demanding settings. They did, however, display the same pattern of activation as the term-born control group, involving particularly the prefrontal and parietal areas and the ACC. The RA revealed that the main difference between the groups occurred when the difficulty of the task was increased. There was no significant group difference in RT.

To our knowledge, there has been no previous study of BOLD activation during a selective attention-working memory task in EPT/ELBW children. Our results are, therefore, unique in showing effect on brain function in a situation requiring simultaneous working memory and selective attention, a common situation that preterm children meet every day. The present study indicates the existence of neuronal markers of selective cognitive impairments at the age of 11 in EPT/ELBW children. The results fit nicely with the few previous fMRI studies of preterm children/adolescents (born $<33 \mathrm{wk}$ ) looking at response inhibition (22), language $(13,16,17,23)$, visual and auditory tasks $(14,24)$, and verbal fluency (25), which also show reduced activation in preterm as compared with term-born children.

Nosarti et al. (22) studied response inhibition in 8 preterm and 14 control adolescents, and found reduced activation in 


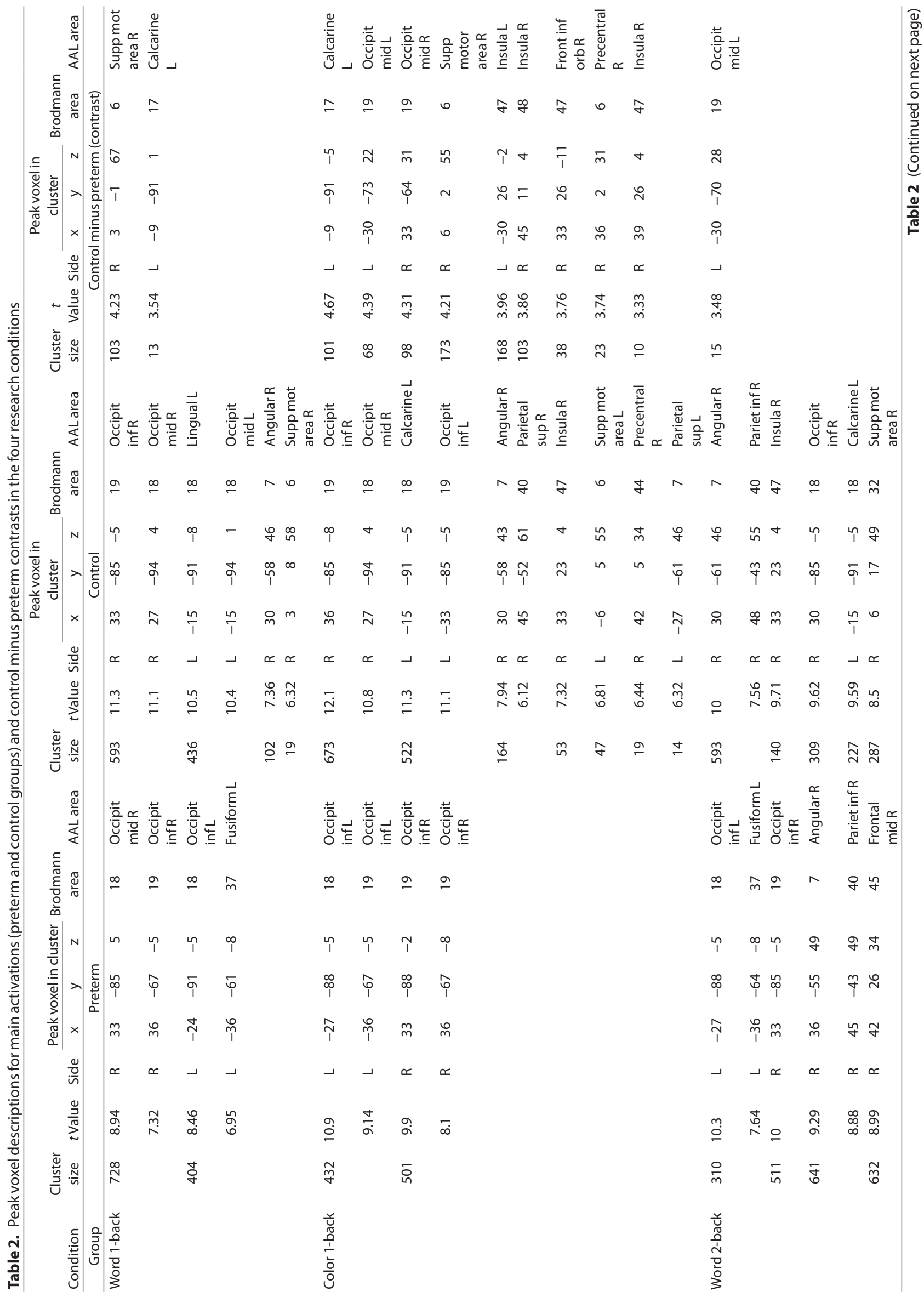




\section{Articles $\mid$ Grifiths et al.}

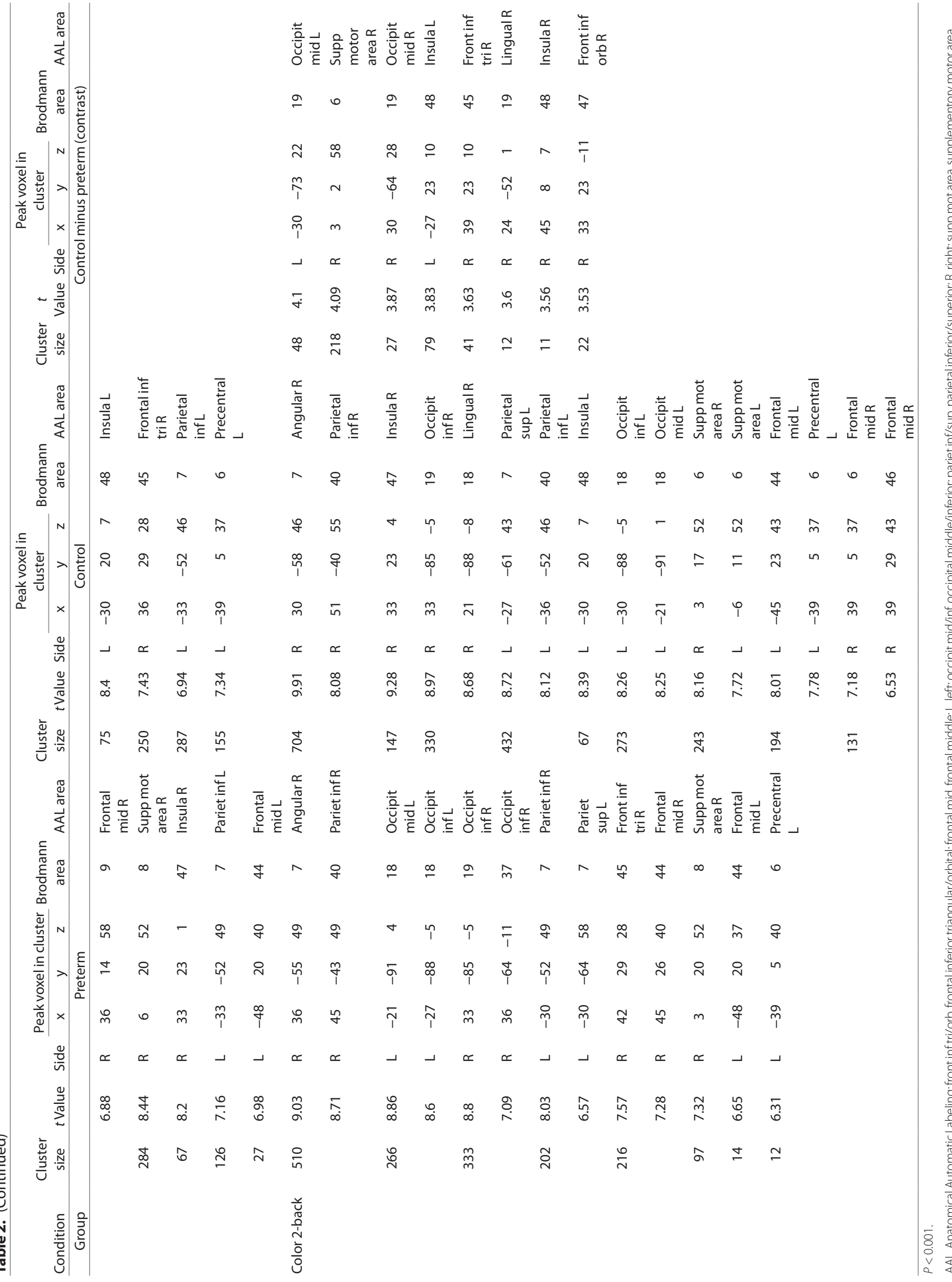


a

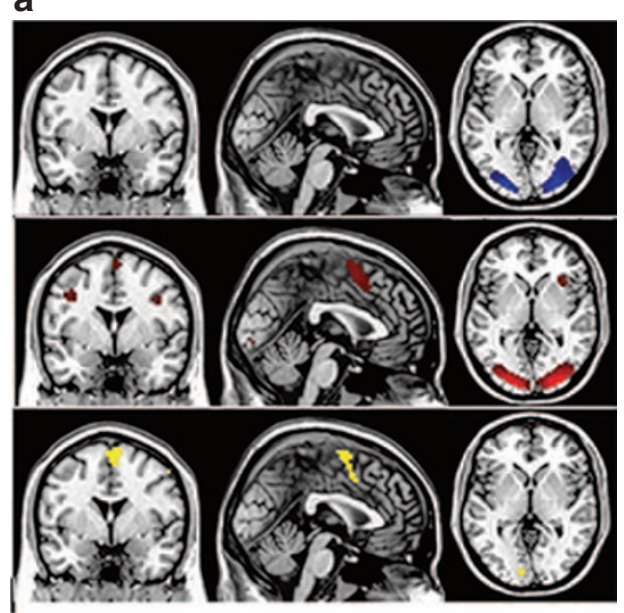

C

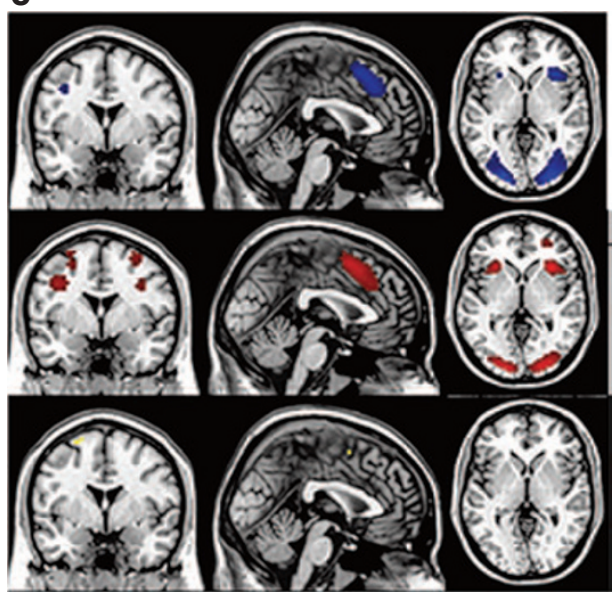

b

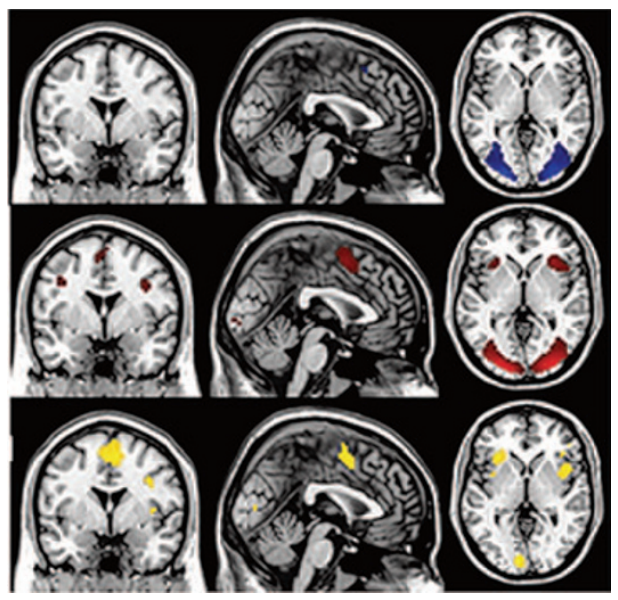

d

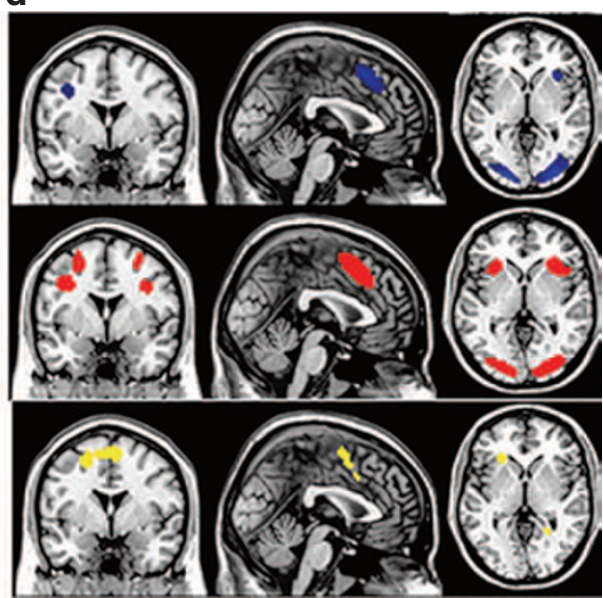

Figure 1. Main BOLD activation patterns for preterm children = blue (FWE corrected; $P<0.05)$, control children $=$ red (FWE corrected; $P<0,05)$, and control children - preterm children contrasts = yellow (uncorrected; $P<0.001$ ) for the four conditions (a) word 1-back, (b) color 1-back, (c) word 2-back, and (d) color 2-back. BOLD, blood oxygen level-dependent; FWE, family-wise error.

the preterm group in the ACC and prefrontal areas as in the present study. Given that response inhibition is also argued to be one of the factors underlying the Stroop interference effect, this may have implications for other executive functions in preterm children $(26,27)$. The additional ROI analysis of the ACC in our study confirmed findings from the main analyses that subtracting activation in the preterm children from that in the control children yielded activation in ACC in both color conditions. This indicates that preterm children rely less on this area than do control children when trying to inhibit an automatic response in the color condition (shift attention from the word cue to the color cue); this effect is intensified when working memory demands are increased as in the 2-back condition. The ACC region is known to be responsive to cognitive load (26), and reduced activation in this area is found in children with attention-deficit hyperactivity disorder (28) and autism spectrum disorders (29) as compared with control children. On the basis of the present findings and previous studies, we suggest that the ACC is a critical area involved in working memory and selective attention, and that our EPT group displayed reduced activation in this important area. Reduced working memory capacity may explain some of the clinical traits that are seen in preterm children, including reduced attention span, memory capacity, and attention $(5,7)$.

Although EPT/ELBW children without major neurodevelopmental disabilities seemingly manage quite well cognitively in everyday life, differences between these children and fullterm controls emerged as the cognitive demands of the experimental tasks increased. This observation is similar to findings in clinical behavioral studies but has not previously been demonstrated by BOLD activation patterns (4-6).

Recent studies have reported increased activation in areas unique to preterm children as compared with controls, and a suggested interpretation has been that they have developed alternative networks $(16,17,22)$. We did not find evidence of increased activation in alternative networks in our preterm group. This may be a consequence of our paradigm not being demanding enough to elicit the use of alternative pathways, or because no alternative pathway was developed for this kind of task, perhaps because they were too young for alternative pathways to have been established. We did consider using a 2- and 3-back version of the n-back task, instead of 1- and 2-back, 


\section{Articles | Griffiths et al.}
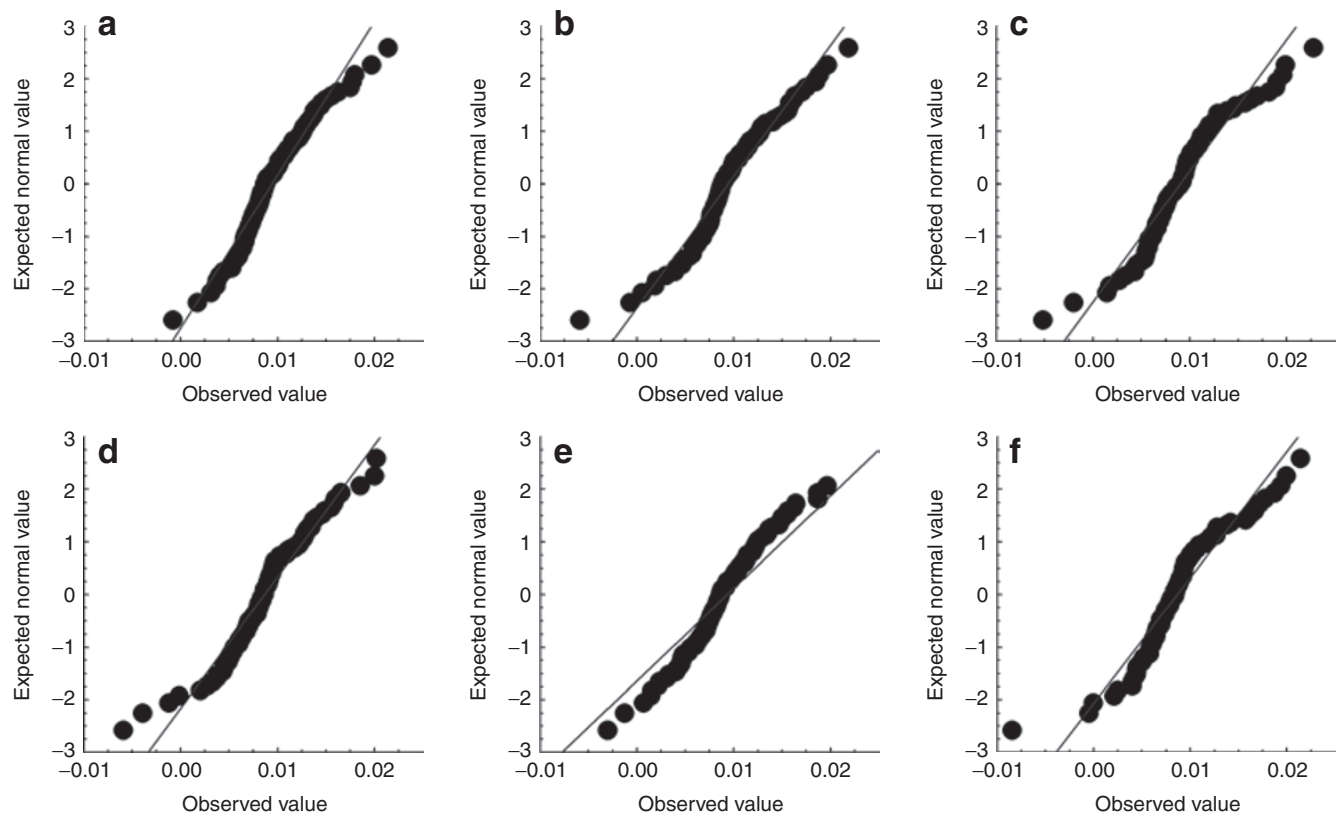

Figure 2. Probability-probability plots of observed vs. theoretical cumulative distribution of the three selected ROIs for (a) control children: occipital middle left, (b) control children: occipital inferior right, (c) control children: supplementary motor area right, (d) EPT/ELBW children: occipital middle left, (e) EPT/ELBW children: occipital inferior right, and (f) EPT/ELBW children: supplementary motor area right. EPT/ELBW, extremely preterm/extremely lowbirth-weight; ROI, region of interest.

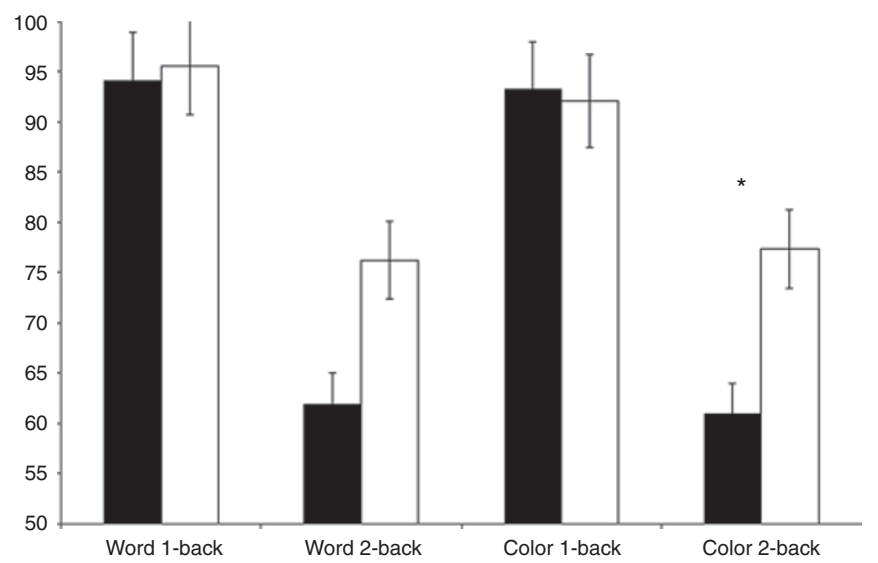

Figure 3. Mean percentage response accuracy (RA) with SEM (vertical thin bars) for the preterm (black) and control (white) group in the four experimental conditions. ${ }^{*} P<0.05$.

but this was abandoned because of the risk of floor effects to a very complicated task in children with a cognitive impairment. We do find it unlikely that the 2-back condition was too easy for the participants because the prescanning instructions and the results indicated that they found the 2-back condition quite difficult. This was also tested in our five pilot scans before starting the study.

The reduced RA in the preterm group as compared with the control group for the 2-back conditions is consistent with previous findings (4). Our sample of EPT/ELBW children was extracted from a larger cohort that had lower intelligent quotient scores at $5 \mathrm{y}$ than expected for children born at term (6). The children in the preterm group displayed slightly longer

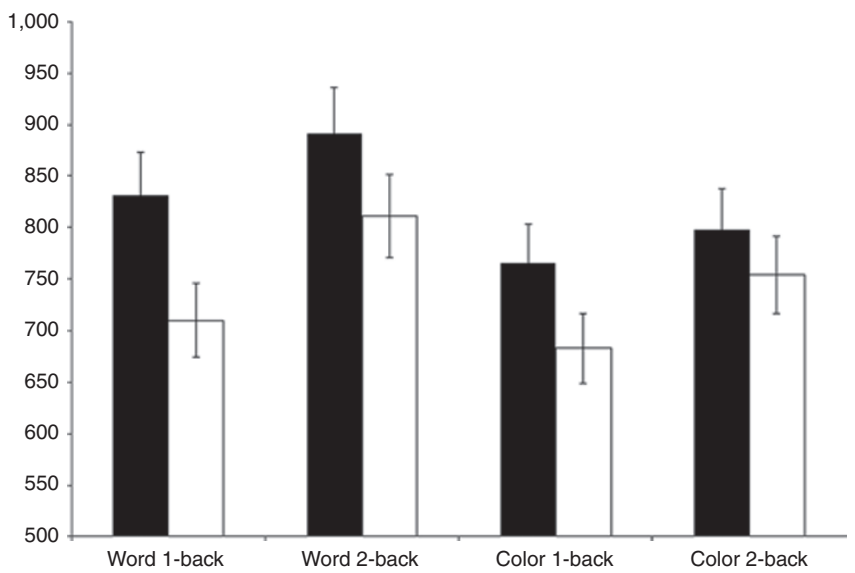

Figure 4. Reaction time (RT) in milliseconds (ms) with SEM (thin vertical bars) for correct answers in the preterm (black) and control (white) groups in the four experimental conditions. No significant differences were found between the groups.

RTs than the control children, but not enough to obtain a significant difference. The significantly reduced RA for this group cannot be explained by inattentiveness because it took them just as long to process the stimuli as the control children. We would have expected RTs to be shorter if they were answering randomly.

Other studies have found significantly slower RT on neuropsychological tests in children with birth weights below $1,500 \mathrm{~g}$ as compared with controls (30). There is general consensus that children born preterm are more likely to have attention deficit problems than children born at term (for review see refs. 4,5,7). Recent studies suggest that lack of attention in only 


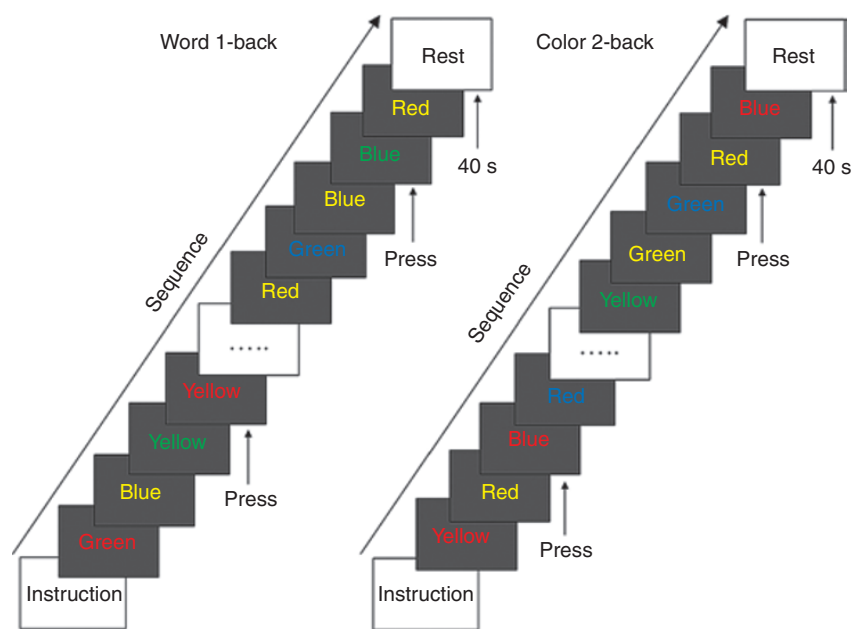

Figure 5. Explanation of task instructions: In the word 1-back condition the child was instructed to press the response button when the written word presented was the same as the stimulus presented one screen back in the presentation sequence. In the color 2-back condition, the response was based on the ink color of the stimuli being the same as the one presented two screens back in the presentation sequence.

a few responses may be the cause of the overall prolonged RTs documented in children with attention-deficit hyperactivity disorder $(31,32)$. This was a relevant possibility also for our preterm children, and a reason to add RT to our study design. Our data do not, however, support that notion, given that the results showed overall similar/slightly increased RTs in EPT/ ELBW children as compared with controls, not a few long answers. Mulder et al. (33) reported slow processing speed to explain reduced academic attainment in very preterm children, and in adults born with birth weights below 1,500 g, RTs were found to be slower than in control participants (30). It is possible that the number of participants in our study was too low to confirm such group differences.

One of the main strengths of this study is the populationbased study group, which in fMRI studies of preterm children is virtually unique, and the high participation rate. This reduces the risk of effects arising from confounding factors typically found in comparable nonpopulation-based studies with lower sample sizes $(16,17,22)$. The same argument applies to control selection based on time of birth (regardless of socioeconomic status), rather than advertisements (which risks attracting high numbers of well-educated families) or classmates (which will be influenced by geographical factors). Because other fMRI studies include both EPT and more mature preterm children, cognitive impairments among participants could be caused by different mechanisms depending on factors such as gestational age and birth weight. We do, however, acknowledge that a larger sample of participants would have increased the generalizability and strength of our results, and would be a future goal, but is still a challenge in fMRI research, given the costs of MRI examinations.

It is known that performance differences may affect the BOLD signal, although the relationship between performance efficacy and BOLD activation is far from known because the data show both increases and decreases in BOLD activation with better performance. This could be a potential limitation of the study. It is, however, unlikely that these effects could be the primary cause of the BOLD activation differences between the groups in the current study because BOLD activation was significantly different for both the color 1-back- and color 2-back conditions, whereas performance differences were significant only for the color 2-back condition. Another limitation is that the uncorrected between-group comparisons remained significant only for the color 1-back condition after false-discovery rate correction, but the main effect of group still showed remaining activations in the same areas as found for an uncorrected threshold. Given the intraindividual variability in BOLD signal in preterm samples, and the fact that the sample sizes were relatively small, it is possible that the data from the preterm children were not normally distributed at each voxel. This would have argued for using a permutation-based approach based on nonparametric tests for significance, rather than a parametric general linear model approach implemented in the SPM8 analysis software $(34,35)$. However, because the between-group normality tests of the BOLD responses in the selected ROIs were not significant, we believe that a difference in error variances between the groups is an unlikely explanation of the results.

In conclusion, using superior methodology to those of previous studies, we have confirmed that children born EPT/ ELBW showed reduced working memory/selective attention capacity for online processing as compared with term controls at the age of $11 \mathrm{y}$. The reduced response accuracies had neuronal correlates with reduced activation in areas responsible for working memory and selective attention.

The knowledge that our EPT/ELBW children did not attain the same level of cognitive control as term-born controls when demands were increased is important for the future of these children, particularly concerning school performance. If teachers know the importance of not giving multiple messages in one go, and of breaking tasks down into smaller parts for these children, this could improve their achievements and self-esteem. We suggest that the neuronal correlates, as identified by fMRI, are important in understanding attention and cognitive impairments in children born preterm and that this knowledge may contribute to improved outcome through alterations in early treatment strategies, particularly in school matters.

\section{METHODS}

\section{Participants}

The present cohort study was part of a Norwegian national followup study of children born EPT (36). The population of children born EPT/ELBW (born before week 28 or birth weight $<1,000 \mathrm{~g}$ ) in 1999 and 2000 in Norway (373 of 638 alive at $2 \mathrm{y}$ of age) were invited to participate at the child's $11 \mathrm{y}$. From this national cohort, the EPT/ ELBW children born in a region in West Norway (Hordaland and Sogn-og Fjordane counties) were invited to take part in an fMRI/ MRI examination. This region has $\sim 10 \%$ of the Norwegian population, and demographic characteristics (e.g., proportions of urban and rural population) are similar to those of Norway as a whole. The regional cohort included $40 \mathrm{EPT} / \mathrm{ELBW}$ children and had previously 
been invited at 2 and $5 \mathrm{y}$ of age for medical and neurodevelopmental examinations (6). Two subjects could not be traced, one was excluded due to extensive neurological disabilities, and one was excluded due to dental braces that caused artifacts in the EPI images. The remaining 36 children had fMRI scans.

For each preterm child, the next term-born child (gestational age $>36 \mathrm{wk}$ ) of the same gender with a birth weight above the 10th percentile $(>3,000 \mathrm{~g})$ and born at the only obstetric department in Hordaland county (Haukeland University Hospital, where all the preterm children were born) was asked to serve as a control. If they declined, the next child on the list was contacted. Thirty-eight children agreed to participate; two were excluded due to dental braces, and 36 were scanned.

\section{MRI Scanning Parameters}

Anatomical and functional (BOLD) MRI data were acquired on a GE Signa HD 1.5 T (Milwaukee, WI) MRI scanner from October 2010 to July 2011. A fast spoiled gradient inversion recovery pulse sequence with standard acquisition parameters was used for the anatomy images, and an EPI sequence was used for the BOLD responses. The parameters of the EPI sequence were TR $3000 \mathrm{~ms}$, matrix $64 \times 64$, FOV $240 \mathrm{~mm}$, no. of slices 28 , slice thickness $5 \mathrm{~mm}$, no gap, and voxel size $3.75 \times 3.75 \times 5 \mathrm{~mm}$. Fourteen EPI scans/blocks (making a total of 112 scans) and 5 initial dummy scans were discarded before the data were analyzed. Total scan time was $\sim 45 \mathrm{~min}$.

\section{Working Memory-Selective Attention Task}

A working memory-selective attention task with single-trial presentations of Stroop color-words in an n-back setting was used $(37,38)$. The task was to remember either a word, independent of the color of ink in which it was written, or the ink color independent of the actual word displayed. Participants were requested to press a button held in their dominant hand when a word or ink color matched that presented either 1- or 2-stimuli backward in the sequence. There were four different conditions (word 1-back, word 2-back, color 1-back, and color 2-back). In the word 1-back and 2-back conditions the participants were told to press the response button when the current stimulus word was the same as the word presented one or two words back in the stimulus sequence (Figure 5). For the color 1-back and 2-back conditions the subjects were asked to respond to the ink color of the words. The selective attention-working memory task was based on increasing levels of cognitive load in two ways: from the 1-back to the 2-back condition and from the monitoring of the word itself to the ink color of the word $(37,39)$. The stimulus sequence is exemplified in Figure 5.

The children were introduced to the procedure through a short computer program test sampling all four research conditions $15 \mathrm{~min}$ in advance of the actual scanning. This also worked as a quick screening for word recognition and possible color blindness that could otherwise interfere with their ability to respond to the correct stimulus. Prior to each of the four conditions, the participants were instructed through headphones which condition was coming next.

The fMRI examination consisted of four runs of the words RED, BLUE, GREEN, and YELLOW, each written in the three incongruent colors (e.g., red written in blue, green, or yellow), making a total of 12 color-word stimuli.

The order of presentation of the four different experimental conditions was counterbalanced in four groups ((i) color 1-back, word 1-back, color 2-back, and word 2-back; (ii) word 1-back, color 1-back, word 2-back, and color 2-back; (iii) color 2-back, word 2-back, color 1-back, and word 1-back; and (iv) word 2-back, color 2-back, word 1-back, and color 1-back), so that one-quarter of the subjects was randomly assigned to one of the four different presentation iterations. This was done in order to avoid any order effects. The stimuli were presented through LCD goggles (Nordic Neurolabs, Bergen, Norway) mounted on the head coil. For each run, there were four ON blocks (A), for which a sequence of 16 stimuli were presented to the participants, and four OFF blocks (B) with no target stimuli, presented serially (A-B-A-B...). In each $\mathrm{ON}$ block, there were three to five target stimuli randomly presented, for which the subject was preinstructed to press the responsekey. Each stimulus was presented for $2.25 \mathrm{~s}$ followed by a blank interval of $0.3 \mathrm{~s}$. The total time for each ON and OFF block was $40.8 \mathrm{~s}$.

\section{fMRI Data Analysis}

SPM8 was used to analyze the BOLD fMRI data. Default preprocessing routines, as implemented in SPM8, were followed for coregistration, realignment, smoothing ( $8 \mathrm{~mm}$ kernel), and normalization (resized voxels $3 \times 3 \times 3 \mathrm{~mm}$ ), with default values for high-pass filters $(128 \mathrm{~Hz})$ and no global scaling. Main effects were family-wise error corrected, the significance level was set to $P<0.05$, and minimum cluster size was set to 10 voxels. Additionally, as family-wise error significance testing was strict to reveal any group differences, false discovery rate significance thresholds were applied. The data were then subjected to first- and second-level significance testing using a 2 (groups: preterm vs. control) $\times$ 2 (load: 1- vs. 2-back) $\times 2$ (instruction: word vs. color) factorial design (ANOVA) and analyzing simple main effects with post-hoc Tukey tests for selected ON-OFF block contrasts, specified for the instruction and load factors, and for the corresponding breakdown of these factors into simple effects. For the control minus preterm and preterm minus control BOLD contrasts, an uncorrected $(P<0.001)$ significance threshold was applied with a minimum cluster size of 10 voxels. The MRICron software (Neuroimaging Informatics Tools and Resources Clearinghouse, Washington, DC) was used to identify the anatomical location of significantly activated clusters, yoking the Anatomical Automatic Labeling atlas (40) and the Brodmann atlas templates.

The ACC is known to be a particularly important area concerning attention, executive functions, and cognitive control $(20,26)$. This region was therefore chosen for a ROI analysis in which only the ACC was analyzed and compared in the two groups (MARINA software, masks for region of interest analysis, version 0.6.1; Bender Institute of Neuroimaging, Giessen, Germany). Significance level was set to $P<$ 0.01 and minimum cluster size was set to 10 voxels.

Informed written consent from the parents and oral consent from the children were collected. The project was approved by the Regional Ethics Committee for Medical Research in western Norway (REKVest \#2009/2271).

\section{ACKNOWLEDGMENTS}

We thank the magnetic resonance imaging staff at the Haraldsplass Hospital for help during the data collecting process, Anne Marie Rød and Alex Craven (Bergen fMRI Group) for help with statistics, Alan Beaton (University of Swansea, Wales) for advice on statistics and writing, and all the extremely preterm/extremely low-birth-weight and control children and their families for participating in the study.

\section{STATEMENT OF FINANCIAL SUPPORT}

This study was financially supported by the Norwegian Extra Foundation for Health and Rehabilitation through the Premature Society, Helse Vest Hospital Trust, and by funding from the Norwegian Western Health Authorities (Helse-Vest) to the Bergen fMRI Group.

Disclosure: K.H. owns stock in Nordic Neurolabs, which produces and sells the LCD goggles used in the study. The other authors declared no conflict of interest.

\section{REFERENCES}

1. Robertson CM, Watt MJ, Dinu IA. Outcomes for the extremely premature infant: what is new? And where are we going? Pediatr Neurol 2009;40:189-96.

2. Moster D, Lie RT, Markestad T. Long-term medical and social consequences of preterm birth. N Engl J Med 2008;359:262-73.

3. Johnson S, Hennessy E, Smith R, Trikic R, Wolke D, Marlow N. Academic attainment and special educational needs in extremely preterm children at 11 years of age: the EPICure study. Arch Dis Child Fetal Neonatal Ed 2009;94:F283-9.

4. Bhutta AT, Cleves MA, Casey PH, Cradock MM, Anand KJ. Cognitive and behavioral outcomes of school-aged children who were born preterm: a meta-analysis. JAMA 2002;288:728-37.

5. Hack M. Adult outcomes of preterm children. J Dev Behav Pediatr 2009;30:460-70.

6. Leversen KT, Sommerfelt K, Rønnestad A, et al. Prediction of neurodevelopmental and sensory outcome at 5 years in Norwegian children born extremely preterm. Pediatrics 2011;127:e630-8. 
7. Shum D, Neulinger K, O'Callaghan M, Mohay H. Attentional problems in children born very preterm or with extremely low birth weight at 7-9 years. Arch Clin Neuropsychol 2008;23:103-12.

8. Anderson PJ, De Luca CR, Hutchinson E, Spencer-Smith MM, Roberts G, Doyle LW; Victorian Infant Collaborative Study Group. Attention problems in a representative sample of extremely preterm/extremely low birth weight children. Dev Neuropsychol 2011;36:57-73.

9. Jongbloed-Pereboom M, Janssen AJ, Steenbergen B, Nijhuis-van der Sanden MW. Motor learning and working memory in children born preterm: a systematic review. Neurosci Biobehav Rev 2012;36:1314-30.

10. Baron IS, Kerns KA, Müller U, Ahronovich MD, Litman FR. Executive functions in extremely low birth weight and late-preterm preschoolers: effects on working memory and response inhibition. Child Neuropsychol 2012;18:586-99.

11. Baddeley A. Working memory: looking back and looking forward. Nat Rev Neurosci 2003;4:829-39.

12. Landrø NI, Rund BR, Lund A, et al. Honig's model of working memory and brain activation: an fMRI study. Neuroreport 2001;12:4047-54.

13. Schafer RJ, Lacadie C, Vohr B, et al. Alterations in functional connectivity for language in prematurely born adolescents. Brain 2009;132(Pt 3):66170 .

14. Gozzo Y, Vohr B, Lacadie C, et al. Alterations in neural connectivity in preterm children at school age. Neuroimage 2009;48:458-63.

15. Curtis WJ, Zhuang J, Townsend EL, Hu X, Nelson CA. Memory in early adolescents born prematurely: a functional magnetic resonance imaging investigation. Dev Neuropsychol 2006;29:341-77.

16. Peterson BS, Vohr B, Kane MJ, et al. A functional magnetic resonance imaging study of language processing and its cognitive correlates in prematurely born children. Pediatrics 2002;110:1153-62.

17. Ment LR, Peterson BS, Vohr B, et al. Cortical recruitment patterns in children born prematurely compared with control subjects during a passive listening functional magnetic resonance imaging task. J Pediatr 2006;149:490-8.

18. Mullen KM, Vohr BR, Katz KH, et al. Preterm birth results in alterations in neural connectivity at age 16 years. Neuroimage 2011;54:2563-70.

19. Cabeza R, Nyberg L. Imaging cognition II: An empirical review of 275 PET and fMRI studies. J Cogn Neurosci 2000;12:1-47.

20. MacLeod CM, MacDonald PA. Interdimensional interference in the Stroop effect: uncovering the cognitive and neural anatomy of attention. Trends Cogn Sci (Regul Ed) 2000;4:383-91.

21. Levene H. Robust test for equality of variances. In: Contributions to Probability and Statistics: Essays in Honour of Harold Hoteling. Palo Alto: Stanford University Press, 1960:278-92.

22. Nosarti C, Rubia K, Smith AB, et al. Altered functional neuroanatomy of response inhibition in adolescent males who were born very preterm. Dev Med Child Neurol 2006;48:265-71.

23. Myers EH, Hampson M, Vohr B, et al. Functional connectivity to a right hemisphere language center in prematurely born adolescents. Neuroimage 2010;51:1445-52.
24. Santhouse AM, Ffytche DH, Howard RJ, et al. The functional significance of perinatal corpus callosum damage: an fMRI study in young adults. Brain 2002;125(Pt 8):1782-92.

25. Rushe TM, Temple CM, Rifkin L, et al. Lateralisation of language function in young adults born very preterm. Arch Dis Child Fetal Neonatal Ed 2004;89:F112-8.

26. Bush G, Luu P, Posner MI. Cognitive and emotional influences in anterior cingulate cortex. Trends Cogn Sci (Regul Ed) 2000;4:215-22.

27. Westerhausen R, Kompus K, Hugdahl K. Impaired cognitive inhibition in schizophrenia: a meta-analysis of the Stroop interference effect. Schizophr Res 2011;133:172-81.

28. Liotti M, Pliszka SR, Perez R, Kothmann D, Woldorff MG. Abnormal brain activity related to performance monitoring and error detection in children with ADHD. Cortex 2005;41:377-88.

29. Agam Y, Joseph RM, Barton JJ, Manoach DS. Reduced cognitive control of response inhibition by the anterior cingulate cortex in autism spectrum disorders. Neuroimage 2010;52:336-47.

30. Strang-Karlsson S, Andersson S, Paile-Hyvärinen M, et al. Slower reaction times and impaired learning in young adults with birth weight $<1500 \mathrm{~g}$. Pediatrics 2010;125:e74-82.

31. Hervey AS, Epstein JN, Curry JF, et al. Reaction time distribution analysis of neuropsychological performance in an ADHD sample. Child Neuropsychol 2006;12:125-40.

32. Epstein JN, Langberg JM, Rosen PJ, et al. Evidence for higher reaction time variability for children with $\mathrm{ADHD}$ on a range of cognitive tasks including reward and event rate manipulations. Neuropsychology 2011;25:427-41.

33. Mulder H, Pitchford NJ, Marlow N. Processing speed and working memory underlie academic attainment in very preterm children. Arch Dis Child Fetal Neonatal Ed 2010;95:F267-72.

34. Hayasaka S, Nichols TE. Validating cluster size inference: random field and permutation methods. Neuroimage 2003;20:2343-56.

35. Thirion B, Pinel P, Mériaux S, Roche A, Dehaene S, Poline JB. Analysis of a large fMRI cohort: Statistical and methodological issues for group analyses. Neuroimage 2007;35:105-20.

36. Markestad T, Kaaresen PI, Rønnestad A, et al.; Norwegian Extreme Prematurity Study Group. Early death, morbidity, and need of treatment among extremely premature infants. Pediatrics 2005;115:1289-98.

37. Stroop J. Studies of interference in serial verbal reactions. J Exp Psychol 1935;18:643-62.

38. Braver TS, Cohen JD, Nystrom LE, Jonides J, Smith EE, Noll DC. A parametric study of prefrontal cortex involvement in human working memory. Neuroimage 1997;5:49-62.

39. Peterson BS, Skudlarski P, Gatenby JC, Zhang H, Anderson AW, Gore JC. An fMRI study of Stroop word-color interference: evidence for cingulate subregions subserving multiple distributed attentional systems. Biol Psychiatry 1999;45:1237-58.

40. Tzourio-Mazoyer N, Landeau B, Papathanassiou D, et al. Automated anatomical labeling of activations in SPM using a macroscopic anatomical parcellation of the MNI MRI single-subject brain. Neuroimage 2002;15:273-89. 